\title{
Data misses dramatic fall in access during COVID-19
}

The British Dental Association has warned the latest NHS Dental Statistics do not fully show the unprecedented fall in access driven by the COVID-19 pandemic.

While limited figures have been offered for the period up to 30 June 2020, data focuses on treatment delivered by high street NHS dentists in England in between 31 March 2019 and 31 March 2020. In this period only $58 \%$ of children had attended an

NHS dentist in the previous 12 months, and $49.6 \%$ of adults in the last two years.

Freedom of information requests by the BDA indicate access to the Urgent Dental Care Network - set up to treat emergencies during lockdown when face-to-face care was suspended in practice - amounted to little over $2 \%$ of normal levels of activity. Paperwork lodged by dentists with the NHS Business Services Authority indicate just 83,300 courses of treatment were delivered in May 2020, compared to an average of around 3.5 million per month in January to March.

The BDA has warned that England has not yet turned the page on low levels of access. Recent BDA surveys indicate the overwhelming majority of practices are now operating at less than a quarter of their former capacity following the resumption of face-to-face care on 8 June.

According to NHS data 38.4 million courses of treatment were delivered last year, an average of around 9.6 million per quarter. Low levels of capacity mean it will be a major challenge to deliver anything resembling those levels going forward.

Practices are facing significant barriers to expanding capacity, including the need to leave surgeries fallow for 60 minutes after an aerosol generating procedure. Unless regulations evolve, the BDA has warned tens of millions of patients in England will effectively lose access to dental services.

Dave Cottam, Chair of the British Dental Association's General Dental Practice Committee said: 'This is data is from another era. Since March patient access has fallen off a cliff, and there is no certainty when or if it can be restored.

'Access was in bad place pre-pandemic. We should lament how few children and adults made it to an NHS dentist last year, but the real question now is how we can even bring the service back to these levels.

'We have practices struggling, and tens of millions of patients need somewhere to go. We need government to work with us to rebuild capacity.'

\section{Tooth extraction crisis will go from bad to worse}

Multiple warnings have been sounded after the latest figures on tooth extractions for children suggests matters will only get worse, as the pandemic exacerbates deep oral health inequalities.

New analysis of official data from the Local Government Association shows 45,000 hospital operations took place to remove rotten teeth in children and teenagers in England in 2018/19 - equating to 177 a day costing the NHS $£ 41.5$ million a year.

Data shows 44,685 extractions of multiple teeth were performed on under-18s in England in 2018/19, an increase of 17 per cent compared to the 38,208 extractions in 2012/13, which cost $£ 27.4$ million.

Levels of health inequality in England were already stark. The latest Public Health
England survey of oral health of five year olds showed a ten-fold difference in severity of dental decay between those in more and less deprived local authority areas. The BDA has said the pandemic will increase this already unacceptable disparity, thanks to lockdown diets, the suspension of public health programmes working on the prevention of tooth decay, and with high street services currently running at less than a quarter of their former capacity.

Tooth decay has been the number one reason for hospital admissions among young children.

Cllr Ian Hudspeth, Chairman of the Local Government Association's Community Wellbeing Board, said: 'These latest figures demonstrate the damage which can be done to young people's teeth through too much sugar intake.

'The fact that, due to the severity of the decay, 177 operations a day to remove multiple teeth in children and teenagers have to be done in a hospital is concerning and also adds to current pressures on the NHS.

'We need to do all we can to reduce how much sugar our children eat and drink, including investing in oral health education so that everyone understands the impact of sugar on teeth and the importance of a good oral hygiene regime.

'Untreated dental care remains one of the most prevalent diseases affecting children and young people's ability to speak, eat, play and socialise.' 